\title{
Impacto de un programa de reducción de infección asociada a catéter en 9 unidades de cuidado intensivo pediátrico en Argentina
} Impact of a program aimed at reducing catheter-related infections implemented in nine pediatric intensive care units in Argentina

\author{
Dra. Ana M. Lenz ${ }^{a}$ Lic. Elena Andion", Dra. Silvina Ruvinsky ${ }^{a}$ Dra. Clarisa Aguirre, \\ Dra. Julieta Álvarez Parmac, Dr. Facundo Jorro ${ }^{d}$, Dra. Gabriela López Cruze, \\ Dr. Martín Pascutto ${ }^{a}$ Dr. Matías Penazzif, Dra. Silvia Santos ${ }^{g}$, \\ Dra. Marcela Zuázaga a Lic. Norma Aquino , Dr. Juan C. Vassallo ${ }^{a}$ \\ Dr. Guillermo Moreno ${ }^{a}$, Dr. Ricardo Magliola ${ }^{a}$, Dr. Aldo Haimovich ${ }^{a}$, \\ Dr. Luis Landrya y Dra. Rosa Bologna ${ }^{a}$
}

a. Hospital de Pediatría "J. P. Garrahan", Ciudad Autónoma de Buenos Aires.

b. Hospital Juan Pablo II, Corrientes.

c. Hospital Interzonal de Niños "Eva Perón", Catamarca.

d. Hospital de Niños "Pedro de Elizalde", Ciudad Autónoma de Buenos Aires.

e. Centro Provincial de Salud Infantil "Eva Perón" (CEPSI), Santiago del Estero.

f. Hospital de Niños de San Justo, Prov. de Buenos Aires.

g. Hospital Regional de Agudos "Eva Perón" (ex Castex), San Martín, Prov. de Buenos Aires.

Correspondencia: Dra. Ana M. Lenz: lenzanamiriam@gmail. com

Financiamiento:

Este trabajo fue parcialmente

financiado por la Beca

Ramón Carrillo-Arturo

Oñativia 2011.

Conflicto de intereses:

Ninguno que declarar.

Recibido: 31-1-2017

Aceptado: 22-8-2017

\section{RESUMEN}

Las infecciones asociadas a catéteres (IAC) conllevan elevada morbimortalidad, con el aumento del uso de recursos hospitalarios. Objetivo. Describir los resultados de un programa para disminuir la tasa de IAC en las unidades de cuidados intensivos pediátricos de Argentina.

Población y métodos. Estudio colaborativo multicéntrico, clínico-epidemiológico, cuasi experimental, de intervención antes y después. Se incluyen niños con catéter venoso central internados en 9 unidades de cuidados intensivos pediátricos de la Ciudad Autónoma de Buenos Aires, conurbano y otras provincias desde junio de 2011 a abril de 2012. Se implementó un paquete de medidas basado en la educación del personal de salud para inserción de catéteres e higiene de manos y uso de listas de verificación con monitoreo de las medidas implementadas. Se compararon el número y la tasa anual de IAC y la tasa de uso de catéter venoso central previa y posterior a la implementación del programa (Stata 8.0).

Resultados. El total de IAC preintervención fue de 117 vs. 74 en el pos. La tasa previa fue 8,6/1000 días de uso y la posintervención, de 5,8/1000 días, RR 0,82 (IC 95\%: 0,68-0,98), p=0,015. La tasa de uso de catéter venoso central se redujo de $54 \%$ a $49 \%$, diferencia no significativa.

Conclusiones. El programa logró un descenso significativo de las tasas de IAC. A partir de él, se implementó la vigilancia de las IAC en todas las unidades de cuidados intensivos pediátricos participantes. La educación y la vigilancia continua son necesarias para mantener y mejorar los resultados alcanzados.

Palabras clave: infecciones relacionadas con catéteres, catéteres venosos centrales, unidades de cuidado intensivo pediátrico.

http: / / dx.doi.org/10.5546/ aap.2018.93

Texto completo en inglés:

http: / / dx.doi.org/10.5546/ aap.2018.eng.93
Cómo citar: Lenz AM, Andion E, Ruvinsky S, et al. Impacto de un programa de reducción de infección asociada a catéter en 9 unidades de cuidado intensivo pediátrico en Argentina. Arch Argent Pediatr 2018;116(2):93-97.

\section{INTRODUCCIÓN}

Los catéteres endovasculares constituyen un dispositivo seguro y de uso rutinario en pacientes críticos, ya que permiten la infusión de inotrópicos, fármacos en altas concentraciones, hemoderivados y el monitoreo hemodinámico. Lamentablemente, su uso no está exento de riesgos, en especial, en los niños pequeños, los cuales suelen reunir casi todos los factores de riesgo. Las infecciones del torrente sanguíneo asociadas a un catéter vascular central (IAC) son un problema de salud relevante, pues representan un aumento de la morbimortalidad en Terapia Intensiva Pediátrica con prolongación en el tiempo de hospitalización de 10 a 40 días y el consecuente incremento de los costos en la atención de pacientes. Es una de las causas más comunes de bacteriemia nosocomial, con rangos que oscilan entre 4 y 8 infecciones por 1000 días-catéter o del 1\% al 13\%, con un rango mayor de infección en las vías de uso intensivo. ${ }^{1-3}$ Se debe considerar la IAC como un problema de seguridad de pacientes. 
Implementar en forma sistemática una serie de intervenciones reunidas en un Programa de Prevención de la Infección Asociada a Catéter ${ }^{4,5}$ en las unidades de cuidados intensivos pediátricos (UCIP) (MultiCOMBO) es, por lo tanto, una estrategia destinada a mejorar la calidad de atención. ${ }^{6}$ Estos programas "MultiCOMBO" son paquetes de medidas ("bundles") que se implementan en conjunto para lograr el resultado deseado, ya que se ha observado que intervenciones con alto nivel de impacto y evidencia implementadas en forma aislada no consiguen disminuir la tasa de infecciones. ${ }^{7}$ Este sistema se ha implementado con éxito en muchos centros hospitalarios internacionales. ${ }^{6,8}$

Se propone la aplicación multidisciplinaria de paquetes de medidas simples con alto nivel de evidencia ${ }^{9}$ y de reconocido impacto en la eficacia para la reducción de IAC. Dentro de ellas, resalta la educación del personal de salud ${ }^{10}$ y la introducción de nuevas técnicas de monitoreo y retroalimentación de la información. ${ }^{11-13}$

Durante 2008-2009, en las UCIP del Hospital Garrahan, la incidencia de IAC oscilaba entre 9 y 12 por cada 1000 días de uso de catéter venoso central (CVC). ${ }^{14,15}$ A partir de julio de 2010, se implementó un Programa Multidisciplinario de Prevención de la Infección Asociada a Catéter, que permitió la reducción de la tasa de IAC a 5,7 por cada 1000 días de uso. ${ }^{16}$ Considerando que esta problemática es general a todas las UCIP, se planteó extender parte del programa a otras unidades del país, a los fines de diseminar los procesos de mejora en la calidad de asistencia y optimizar la oferta del servicio.

El objetivo de este trabajo es comparar la incidencia de IAC antes y después de la implementación de un programa de paquetes de medidas a tal fin en las diferentes UCIP participantes.

\section{PACIENTES Y MÉTODOS}

Estudio multicéntrico, diseño de tipo antesdespués, cuasiexperimental, que evaluó el impacto sobre la incidencia de IAC antes y después de la implementación del paquete de medidas tendientes a disminuir la IAC.

En el país, existen 132 UCIP, de las cuales el $24 \%$ pertenecen a hospitales públicos. La selección se realizó entre UCIP de hospitales públicos, en función de la participación previa de estas UCIP en un programa de mejora de calidad implementado en los 3 años anteriores y que, además, cumplían requisitos de factibilidad de implementación y registro, aprobación de las autoridades sanitarias jurisdiccionales y de los profesionales a cargo del servicio.

Participaron 9 UCIP de 7 hospitales: Hospital de Pediatría "Juan P. Garrahan", Hospital General de Niños "Pedro de Elizalde", Hospital de Niños de San Justo, Hospital Regional de Agudos "Eva Perón" (ex Castex), Hospital Interzonal de Niños "Eva Perón" de Catamarca, Hospital Pediátrico "Juan Pablo II" de Corrientes, Centro Provincial de Salud Infantil “Eva Perón" (CEPSI) de Santiago del Estero. El protocolo fue aprobado por el Comité de Investigación del Hospital Garrahan (centro coordinador del trabajo multicéntrico) y por los Comités de Investigación de cada uno de los hospitales participantes. No se solicitó consentimiento informado, dado que no se realizaron a los pacientes intervenciones distintas a las utilizadas en la práctica diaria.

Se incluyeron todos los niños con CVC internados en las UCIP de los hospitales participantes entre el 1 de junio de 2011 y el 30 de abril de 2012. Las variables registradas fueron tasa de IAC por 1000 días de CVC según definición de vigilancia epidemiológica del Centers for Disease Control and Prevention (CDC) (véanse las definiciones en el Anexo 1, en formato electrónico), tasa de utilización de CVC, adherencia al lavado de manos (véase Anexo 2, en formato electrónico), adherencia al chequeo de colocación de CVC (véase Anexo 3, en formato electrónico). Para el análisis de la efectividad del programa, se consignó como etapa preintervención el período comprendido entre el 1 de agosto de 2010 y el 31 de mayo 2011 y el período posintervención del 1 de junio de 2011 hasta el 31 marzo de 2012.

Se realizaron mensualmente talleres acerca de higiene de manos y su aplicación de los cinco momentos en el punto de atención del paciente y prevención de infecciones hospitalarias dentro de las UCIP. La evolución y los resultados obtenidos fueron difundidos en forma mensual a todo el personal. A través de reuniones con el grupo ejecutor y de encuentros virtuales con los distintos centros, se discutieron y consensuaron posibles soluciones a los problemas o dudas que fueron surgiendo en el desarrollo del programa. Se utilizó el Programa de Referencia y Contrarreferencia vigente en el Hospital Garrahan, en el marco del Subprograma de Colaboración Docente-Asistencial con las UCIP de los hospitales CEPSI de Santiago del Estero y "Eva Perón” de Catamarca.

En el Anexo 4 (véase en formato electrónico), se describe el programa y las etapas de implementación en los diferentes centros participantes. 


\section{Análisis estadístico}

En este trabajo, se identificaron las variables dependientes como los indicadores de la intervención (tasa de uso de CVC, tasa de IAC por 1000 días-catéter, porcentaje de IAC, número absoluto de IAC, porcentaje de adhesión a las medidas (en forma global o desagregadas por combo), considerando la intervención a los paquetes de medida en su conjunto, y la comparación entre los indicadores seleccionados antes y después de las intervenciones. Las variables continuas fueron resumidas mediante medidas de posición y dispersión acordes a la distribución; los datos categóricos, presentados mediante tabla de frecuencias y gráficos. Para el análisis bivariado entre variables, se usó test de $t$, Wilcoxon o chi cuadrado según correspondiera. El nivel de significación se estableció en $<0,05$; el paquete estadístico para el procesamiento fue Stata 8.0.

\section{RESULTADOS}

En la Tabla 1, se detallan los datos relevados en el período preintervención de cada una de las UCIP participantes. El impacto del programa respecto a la reducción en el número de episodios de infecciones asociadas a CVC (IAC), a la tasa de uso de catéteres y a la tasa de infecciones relacionadas con CVC se detalla en la Tabla 2. En las UCIP de los centros que iniciaron su primer año del programa, se obtuvo una reducción significativa de la tasa de IAC, como así también una reducción del número de episodios totales de IAC respecto al año previo de iniciado el programa. Esto no se observó en las 3 UCIP del centro coordinador que ya habían iniciado el programa un año antes, donde mantuvieron tasas similares. El total de IAC en el período pre- vs. posintervención fue de 117 vs. 74 . (véase la Tabla 2) y la tasa total de IAC fue 8,6 cada
1000 días de uso de CVC y de 5,78 cada 1000 días de CVC en el período pre- vs. posintervención, respectivamente, RR 0,82 (IC 95\%: 0,68-0,98), $\mathrm{p}=0,015$. La tasa de uso de CVC se redujo de $54 \%$ a $49 \%$. Solo 5 UCIP reportaron datos de la adherencia de higiene de manos, por lo que no se consideró útil el análisis de esta variable.

\section{DISCUSIÓN}

La importancia de este trabajo multicéntrico fue demostrar que, con la implementación de programas destinados a disminuir la tasa de infecciones intrahospitalarias, se podían lograr resultados significativos, con medidas simples que estaban al alcance del personal de salud. El pilar de estos resultados está en el compromiso de la institución en llevar a cabo el programa, la educación continua, el cambio de conducta y la toma de conciencia de que no se necesita de mayor tecnología para obtener resultados satisfactorios. Es importante destacar que el programa incluyó la participación de todo el personal que intervenía en el cuidado de los pacientes, no solo médicos y enfermeros, sino técnicos y personal de limpieza. Además, se debe mantener una vigilancia estrecha de los resultados a través de la estadística y compararla en forma temporal con la misma institución y, a su vez, con organismos nacionales e internacionales de vigilancia epidemiológica. ${ }^{15,17}$

Hace más de una década que se comenzó a prestar atención a la educación del personal de salud con respecto a medidas para la prevención de infecciones intrahospitalarias. Coopersmith et al., en 2002, ${ }^{10}$ lograron una disminución de un $66 \%$ de las IAC (de 10,8 a 3,7), en la cual se realizó un pretest y postest al grupo de enfermería de una Unidad de Cuidados Intensivos de adultos sobre el conocimiento en prevención de infecciones; luego se entregó un módulo de 10 páginas con

TABLA 1. Características basales de cada una de las unidades de cuidados intensivos pediátricos participantes en el período preintervención

\begin{tabular}{|c|c|c|c|c|c|c|c|}
\hline UCIP & Capacidad & $\begin{array}{c}\text { Mortalidad } \\
\%\end{array}$ & $\begin{array}{c}\text { Ocupación } \\
\%\end{array}$ & $\begin{array}{c}\mathrm{N}^{\circ} \text { de } \\
\text { pacientes/día }\end{array}$ & $\begin{array}{c}\mathrm{N}^{0} \text { de días } \\
\text { de CVC }\end{array}$ & $\begin{array}{r}\text { Tasa de uso } \\
\text { de CVC/100 }\end{array}$ & $\begin{array}{c}\text { Tasa de } \\
\text { bacteriemia/1000 }\end{array}$ \\
\hline UCIP 1 & 15 camas & 15 & ND & 2667 & 590 & 22,12 & 22 \\
\hline UCIP 2 & 12 camas & 22 & 67 & 2449 & 1510 & 61,6 & 18,54 \\
\hline UCIP 3 & 6 camas & 8,5 & ND & 1700 & 719 & 42 & 6,95 \\
\hline UCIP 4 & 8 camas & 10,8 & 85 & 1486 & 952 & 58 & 9,45 \\
\hline UCIP 5 & 11 camas & 8,58 & 63,6 & ND & 1837 & 64 & 13,6 \\
\hline UCIP 6 & 6 camas & ND & ND & 1257 & 262 & 20,84 & 30,53 \\
\hline UCIP 7 & 18 camas & 6,49 & 85,43 & 5074 & 2740 & 54 & 6,56 \\
\hline UCIP 8 & 21 camas & 6,70 & 91,66 & 4469 & 2816 & 63 & 6,56 \\
\hline UCIP 9 & 18 camas & 9,18 & 84,55 & 4425 & 2167 & 49 & 5 \\
\hline
\end{tabular}

UCIP: Unidad de Cuidados Intensivos Pediátricos; CVC: catéter venoso central; ND: no hay dato. 
información sobre este tema y un entrenamiento en la Unidad de Cuidados Intensivos. Berenholtz et ál., en 2004,7 agregaron al entrenamiento una lista de chequeo en la colocación de catéteres, facultaron a enfermería para interrumpir el procedimiento en caso de romper con la antisepsia y el relevamiento diario de la necesidad de mantener en el paciente el CVC. Este grupo redujo la tasa de infecciones de 11,5/1000 a 0/1000 días-catéter, lo que demostró que la implementación de varias medidas basadas en la evidencia al mismo tiempo tenía gran impacto. En este trabajo, Berenholtz incluyó a un grupo control en otra terapia quirúrgica, en la que la única intervención realizada fue el entrenamiento al equipo de salud, sin las otras medidas de control. La reducción de la tasa de infecciones fue del $75 \%$; comparada con el otro grupo, en el que se habían introducido varias medidas al mismo tiempo, la reducción fue del 100\%. ${ }^{7}$ Estos programas de paquetes de medidas se replicaron en muchos centros con buenos resultados en el descenso de las infecciones intrahospitalarias. ${ }^{18,19}$
La mayoría de los centros que integraron el programa no tenían un sistema de vigilancia activo para el registro de las infecciones hospitalarias en las distintas UCIP, por lo que se entrenó al personal en el registro y armado de las estadísticas. La implementación de listas de verificación para el lavado de manos y la colocación de catéteres tuvo básicamente un impacto educativo, ya que permitió capacitar en las buenas prácticas de la prevención de las infecciones hospitalarias.

Se considera que uno de los aportes más importantes logrado por el programa fue el inicio del sistema de vigilancia activo de las infecciones hospitalarias, inicialmente, de las más frecuentes en población pediátrica, que son las bacteriemias relacionadas con CVC.

En el período posintervención, se observó, en los 6 centros que iniciaban su primer año de programa, una reducción en el número de episodios de IAC y, además, una reducción de la tasa de utilización de CVC, lo que significó que, probablemente en este punto, se hubiera logrado

TABLA 2. Análisis de impacto pre-vs. posintervención del programa

\begin{tabular}{|c|c|c|c|c|}
\hline & & Preintervención & Posintervención & $\mathbf{P}$ \\
\hline UCIP 1 & $\begin{array}{l}\text { No de episodios de IAC } \\
\text { Tasa de uso de CVC } \\
\text { Tasa de IAC x } 1000 \text { días }\end{array}$ & $\begin{array}{c}13 \\
22,12 \% \\
22\end{array}$ & $\begin{array}{c}6 \\
22,87 \% \\
10,13\end{array}$ & $\begin{array}{c}\mathrm{P}=0,5 \\
\mathrm{NS} \\
\mathrm{P}=0,16 \mathrm{RR}=0,63(\mathrm{IC} 95 \%: 0,32-1,1)\end{array}$ \\
\hline UCIP 2 & $\begin{array}{l}\text { No de episodios de IAC } \\
\text { Tasa de uso de CVC } \\
\text { Tasa de IAC x } 1000 \text { días }\end{array}$ & $\begin{array}{c}28 \\
60 \% \\
18,54\end{array}$ & $\begin{array}{c}6 \\
52 \% \\
4,89\end{array}$ & $\begin{array}{c}\mathrm{P}=0,015 \\
\mathrm{NS} \\
\mathrm{p}=0,001 \mathrm{RR}=0,39(\mathrm{IC} 95 \%: 0,19-0,81)\end{array}$ \\
\hline UCIP 3 & $\begin{array}{l}\text { No de episodios de IAC } \\
\text { Tasa de uso de CVC } \\
\text { Tasa de IAC x } 1000 \text { días }\end{array}$ & $\begin{array}{c}5 \\
42 \% \\
6,95 / 1000\end{array}$ & $\begin{array}{c}3 \\
37,8 \% \\
3,2\end{array}$ & $\begin{array}{c}\mathrm{P}=0,015 \\
\mathrm{NS} \\
\mathrm{P}=0,31 \mathrm{RR}=0,67(\mathrm{IC} 95 \%: 0,27-1,64)\end{array}$ \\
\hline UCIP 4 & $\begin{array}{l}\text { No de episodios de IAC } \\
\text { Tasa de uso de CVC } \\
\text { Tasa de IAC x } 1000 \text { días }\end{array}$ & $\begin{array}{c}9 \\
54,5 \% \\
9,45 / 1000\end{array}$ & $\begin{array}{c}1 \\
49 \% \\
0,9\end{array}$ & $\begin{array}{c}\mathrm{P}=0,06 \\
\mathrm{NS} \\
\mathrm{P}=0,007 \mathrm{RR}=0,19(\mathrm{IC} 95 \%: 0,03-0,67)\end{array}$ \\
\hline UCIP 5 & $\begin{array}{l}\text { No de episodios de IAC } \\
\text { Tasa de uso de CVC } \\
\text { Tasa de IAC x } 1000 \text { días }\end{array}$ & $\begin{array}{c}25 \\
61,6 \% \\
13,6 / 1000\end{array}$ & $\begin{array}{c}6 \\
64 \% \\
3,76\end{array}$ & $\begin{array}{c}\mathrm{P}=0,05 \\
\mathrm{NS} \\
\mathrm{P}=0,004 \mathrm{RR}=0,41(\mathrm{IC} 95 \%: 0,20-0,85)\end{array}$ \\
\hline UCIP 6 & $\begin{array}{l}\text { No de episodios de IAC } \\
\text { Tasa de uso de CVC } \\
\text { Tasa de IAC x } 1000 \text { días }\end{array}$ & $\begin{array}{c}8 \\
15,7 \% \\
30,53 / 1000\end{array}$ & $\begin{array}{c}3 \\
37,6 \% \\
8,28\end{array}$ & $\begin{array}{c}\mathrm{P}=0,45 \\
\mathrm{P}<0,001 \mathrm{RR}=1,54(\mathrm{IC} 95 \%: 1,40-1,66) \\
\mathrm{P}=0,06 \mathrm{RR}=0,47(\mathrm{IC} \text { 95\%: 0,18-1,23) }\end{array}$ \\
\hline UCIP 7 & $\begin{array}{l}\text { No de episodios de IAC } \\
\text { Tasa de uso de CVC } \\
\text { Tasa de IAC x } 1000 \text { días }\end{array}$ & $\begin{array}{c}18 \\
53 \% \\
6,56 / 1000\end{array}$ & $\begin{array}{c}18 \\
39 \% \\
8,26\end{array}$ & $\begin{array}{c}\mathrm{P}=0,72 \\
\mathrm{P}<0,001 \mathrm{RR}=0,60(\mathrm{IC} 95 \%: 0,55-0,65) \\
\text { NS }\end{array}$ \\
\hline UCIP 8 & $\begin{array}{l}\text { No de episodios de IAC } \\
\text { Tasa de uso de CVC } \\
\text { Tasa de IAC x } 1000 \text { días }\end{array}$ & $\begin{array}{c}18 \\
61 \% \\
6,50 / 1000\end{array}$ & $\begin{array}{c}16 \\
70,38 \\
8\end{array}$ & $\begin{array}{c}\mathrm{P}=0,34 \\
\mathrm{NS} \\
\mathrm{NS}\end{array}$ \\
\hline UCIP 9 & $\begin{array}{l}\text { No de episodios de IAC } \\
\text { Tasa de uso de CVC } \\
\text { Tasa de IAC x } 1000 \text { días }\end{array}$ & $\begin{array}{c}11 \\
57 \% \\
5 / 1000\end{array}$ & $\begin{array}{c}15 \\
54 \% \\
6,45 / 1000\end{array}$ & $\begin{array}{c}\mathrm{P}=0,68 \\
\mathrm{NS} \\
\mathrm{NS}\end{array}$ \\
\hline
\end{tabular}

UCIP: Unidad de Cuidados Intensivos Pediátricos; CVC: catéter venoso central; IAC: infecciones asociadas a catéteres;

NS: no significativa; IC: intervalo de confianza; RR: riesgo relativo; P: significación. 
en algunos centros una mayor vigilancia de la necesidad de que el paciente no continuara con el CVC colocado más allá de lo estrictamente necesario para su tratamiento. Asimismo, se observaron reducciones muy importantes de la tasa de IAC. En algunos casos, esta reducción fue estadísticamente significativa y, en otros, se vio una tendencia en su significancia, pero como consecuencia de la reducción de los días de CVC, es probable que se sobreestimara el numerador $y$, de esta forma, el resultado de la tasa no lograra alcanzar la significancia estadística esperada.

En las 3 unidades de terapia intensiva pediátrica (UTIP) del centro coordinador, se había observado, en el primer año de iniciado el programa (año 2010), una reducción de alrededor del 50\%.14,16 En este estudio (año 2011), no se observó una disminución respecto a las tasas basales, pero sí su estabilidad. Sin embargo, hay que considerar que este fue el segundo año del programa y es posible que exista aparición de resistencia a las medidas implementadas o menor adherencia por parte del personal de salud. Está descrito que, cuando disminuye la vigilancia y las estrategias para mantener estas medidas, disminuye la adherencia y la tasa de infecciones vuelve a subir. ${ }^{20-22}$ La alta rotación de personal, frecuente en las unidades de cuidados críticos, suma mayores dificultades a este desafío colectivo y hace necesario buscar nuevas estrategias de estímulo de manera permanente con el objetivo de lograr una mayor adherencia. El cambio fundamental consiste en lograr modificaciones en la cultura del personal de salud sobre la importancia de la higiene de manos y el cumplimiento de todos los puntos del programa por parte de todo el personal para alcanzar una reducción sostenida de las infecciones hospitalarias y continuar descendiendo las tasas de IAC actuales.

Como limitaciones del estudio, no se trata de un ensayo clínico controlado, sino de un diseño de tipo antes-después.

Se considera que los resultados obtenidos por el programa muestran evidencia suficiente para que continúe siendo implementado y replicado en las distintas UCIP de nuestro país.

\section{CONCLUSIÓN}

La implementación del programa logró una reducción estadísticamente significativa en la tasa de bacteriemia relacionada con CVC en niños internados en UCIP.

\section{REFERENCIAS}

1. O'Grady N. Alexander M, Dellinger E, et al. Guidelines for the Prevention of Intravascular Catheter-Related Infections. The Hospital Infection Control Practices Advisory
Committee, Center for Disease Control and Prevention, U.S. Pediatrics 2002;110(5):e51.

2. De Cicco L, Schachner B, Giraudo N, et al. Implementación de un Sistema de Vigilancia Activa de Infecciones Intrahospitalarias en una Unidad de Cuidados Intensivos Pediátricos. Arch Argent Pediatr 2005;103(2):118-28.

3. Odetola FO, Moler FW, Dechert RE, et al. Nosocomial catheter - related bloodstream infections in a pediatric intensive care unit: risk and rates associated with various intravascular technologies. Pediatr Crit Care Med 2003 Oct;4(4):432-6.

4. McGeeDC, Gould MK. Preventing Complications of Central Venous Catheterization. N Engl J Med 2003;348(12):1123-33.

5. Pronovost P, Needham D, Berenholtz S, et al. An intervention to decrease catheter-related bloodstream infections in the UCI. N Engl J Med 2006;355(26):2725-32.

6. Costello JM, Morrow DF, Graham DA, et al Systematic intervention to reduce central line-associated bloodstream infection rates in a pediatric cardiac intensive care unit. Pediatrics 2008;121(5):915-23.

7. BerenholtzSM, PronovostPA, Lipsett PA, et al. Eliminating catheter-related bloodstream infections in the intensive care unit. Crit Care Med 2004;32(10):2014-20.

8. Menegueti M, Ardison KM, Bellissimo-Rodrígues F, et al. The Impact of Implementation of Bundle to Reduce Catheter-Related Bloodstream Infection Rates. J Clin Med Res 2015;7(11):857-61.

9. NNISSystem. National Nosocomial Infections Surveillance (NNIS) system report, data summary from January 1990May 1999, issued June 1999. A report from the NNIS System. Am J Infect Control 1999;27(6):520-32.

10. Coopersmith CM, Rebmann TL, Zack JE, et al. Effect of an education program on decreasing catheter-related bloodstream infections in the surgical intensive care unit. Crit Care Med 2002;30(1):59-64.

11. Curtis J, Cook DJ, Wall RJ. et al. Intensive care unit quality improvement: A "how to" guide for the interdisciplinary team. Crit Care Med 2006;34(1):211-8.

12. Pronovost P, Goeschel C, Colantuoni E, et al. Sustaining reductions in catheter related bloodstream infections in Michigan intensive care units: observational study. BMJ 2010;340:c309.

13. Pronovost PJ, Berenholtz SM, Needham DM. Translating evidence into practice: A model for large scale knowledge translation. BMJ 2008;337:a1714.

14. Hospital Garrahan. Vigilancia epidemiológica anual. Comunicación interna. 2010-2012.

15. Dudeck MA, Horan TC, Peterson KD, et al. National Healthcare Safety Network(NHSN) Report. Data Summary for 2009. Device Asociated Module. Am J Infect Control 2011;39(5):349-67.

16. Lenz AM, Latini B, Ruvinsky S. Programa de Prevención de Infecciones Asociadas a Catéteres en UCIP. En VIII Jornadas Multidisciplinarias Hospital Garrahan. 16-30 agosto de 2011; Buenos Aires.

17. Programa Nacional de Epidemiología y Control de Infecciones Hospitalarias (VIHDA). República Argentina. Ministerio de Salud. [Acceso: 22 de agosto de 2017]. Disponible en: www.vihda.gov.ar.

18. Espiau M, Pujol M, Campins-Martí M, et al. Incidencia de bacteriemia asociada a catéter venoso central en una unidad de cuidados intensivos. An Pediatr (Barc) 2011;75(3):188-93.

19. Semelsberger, CF. Educational interventions to reduce the rate of central catheter-related bloodstream infections in the NICU: a review of the research literature. Neonatal Netw 2009;28(6):391-5

20. Hong TS, Bush EC, Hauenstein MF, et al. A hand hygiene compliance check system: brief communication on a system to improve hand hygiene compliance in hospitals and reduce infection. J Med Syst 2015;39(6):69.

21. Gluyas H. Understanding non-compliance with hand hygiene practices. Nurs Stand 2015;29(35):40-6.

22. White KM, Jimmieson NL, Obst PL, et al. Using a theory of planned behaviour framework to explore hand hygiene beliefs at the ' 5 critical moments' among Australian hospitalbased nurses. BMC Health Serv Res 2015;15:59. 


\section{ANEXO 1}

Definiciones

Se utilizó como medida estadística la tasa de infecciones por 1000 días de uso de CVC (recomendado por el CDC). La fórmula era la siguiente:

IAC/1000 días de CVC: Número de bacteriemias en un período x 1000

Número de días de uso de CVC en el mismo período determinado

Otra medida que se usó fue la tasa de utilización de CVC, cuya fórmula era la siguiente:

Tasa de utilización: Número de días de uso de CVC en un período determinado x 100

Número de días totales de internación de pacientes en el mismo período 
ANEXO 2

Planilla de observación para higiene de manos. Aplicación de los cinco momentos en el punto de atención del paciente. Lista de chequeo

\begin{tabular}{|c|c|c|c|c|c|c|}
\hline \multicolumn{5}{|c|}{ Planilla de observación para higiene de manos } & FECHA DE SESIÓN & ........................... \\
\hline \multirow{2}{*}{\multicolumn{2}{|c|}{ OBSERVACIÓN }} & & & & \multicolumn{2}{|c|}{ PROFESIÓN OBSERVADO } \\
\hline & & & & & \multicolumn{2}{|c|}{ ÁREA DE INTERNACIÓN } \\
\hline OPORTUNIDAD & \multicolumn{2}{|c|}{ INDICACIONES } & MODO & OPORTUNIDAD & INDICACIONES & MODO \\
\hline \multirow{5}{*}{1} & $\square$ & $\begin{array}{r}\text { Pre-contacto } \\
\text { con el paciente }\end{array}$ & \multirow{5}{*}{$\underset{\text { Frotado }}{\square}$} & \multirow{5}{*}{2} & $\begin{array}{r}\text { Pre-contacto } \\
\text { con el paciente }\end{array}$ & \multirow{4}{*}{$\begin{array}{l}\square \\
\text { Frotado } \\
\square \\
\text { Lavado }\end{array}$} \\
\hline & & $\begin{array}{r}\text { Pre-tarea } \\
\text { aséptica }\end{array}$ & & & $\begin{array}{r}\text { Pre-tarea } \\
\text { aséptica }\end{array}$ & \\
\hline & & $\begin{array}{l}\text { Post-contacto } \\
\text { idos corporales }\end{array}$ & & & $\begin{array}{l}\text { Post-contacto } \\
\text { fluídos corporales }\end{array}$ & \\
\hline & & $\begin{array}{l}\text { Post-contacto } \\
\text { con el paciente }\end{array}$ & & & $\begin{array}{l}\text { Post-contacto } \\
\text { con el paciente }\end{array}$ & \\
\hline & & $\begin{array}{r}\text { Post-contacto } \\
\text { con el entorno } \\
\end{array}$ & & & $\begin{array}{l}\text { Post-contacto } \\
\text { con el entorno }\end{array}$ & No realizado \\
\hline OPORTUNIDAD & & ICACIONES & MODO & OPORTUNIDAD & INDICACIONES & MODO \\
\hline \multirow{5}{*}{3} & & $\begin{array}{r}\text { Pre-contacto } \\
\text { con el paciente }\end{array}$ & \multirow{5}{*}{$\begin{array}{c}\square \text { Frotado } \\
\text { Lavado } \\
\text { No realizado }\end{array}$} & \multirow{5}{*}{4} & $\begin{array}{r}\text { Pre-contacto } \\
\text { con el paciente }\end{array}$ & \multirow{5}{*}{$\begin{array}{c}\square \text { Frotado } \\
\text { Lavado realizado } \\
\end{array}$} \\
\hline & & $\begin{array}{l}\text { Pre-tarea } \\
\text { aséptica }\end{array}$ & & & $\begin{array}{l}\text { Pre-tarea } \\
\text { aséptica }\end{array}$ & \\
\hline & & $\begin{array}{l}\text { Post-contacto } \\
\text { dos corporales }\end{array}$ & & & $\begin{array}{r}\text { Post-contacto } \\
\text { fluídos corporales }\end{array}$ & \\
\hline & & $\begin{array}{l}\text { Post-contacto } \\
\text { con el paciente }\end{array}$ & & & $\begin{array}{l}\text { Post-contacto } \\
\text { con el paciente }\end{array}$ & \\
\hline & & $\begin{array}{l}\text { Post-contacto } \\
\text { con el entorno }\end{array}$ & & & $\begin{array}{l}\text { Post-contacto } \\
\text { con el entorno }\end{array}$ & \\
\hline OPORTUNIDAD & & ICACIONES & MODO & OPORTUNIDAD & INDICACIONES & MODO \\
\hline \multirow{5}{*}{5} & & $\begin{array}{l}\text { Pre-contacto } \\
\text { con el paciente }\end{array}$ & \multirow{5}{*}{$\begin{array}{c}\square \\
\text { Frotado } \\
\text { No realizado } \\
\square\end{array}$} & \multirow{5}{*}{6} & $\begin{array}{r}\text { Pre-contacto } \\
\text { con el paciente }\end{array}$ & \multirow{5}{*}{$\begin{array}{c}\square \\
\text { Frotado } \\
\square_{\text {Lavado }}^{\square} \\
\square\end{array}$} \\
\hline & & $\begin{array}{r}\text { Pre-tarea } \\
\text { aséptica }\end{array}$ & & & $\begin{array}{r}\text { Pre-tarea } \\
\text { aséptica }\end{array}$ & \\
\hline & & $\begin{array}{l}\text { Post-contacto } \\
\text { ídos corporales }\end{array}$ & & & $\begin{array}{r}\text { Post-contacto } \\
\text { fluídos corporales }\end{array}$ & \\
\hline & & $\begin{array}{l}\text { Post-contacto } \\
\text { con el paciente }\end{array}$ & & & $\begin{array}{l}\text { Post-contacto } \\
\text { con el paciente }\end{array}$ & \\
\hline & & $\begin{array}{l}\text { Post-contacto } \\
\text { con el entorno }\end{array}$ & & & $\begin{array}{l}\text { Post-contacto } \\
\text { con el entorno }\end{array}$ & \\
\hline OPORTUNIDAD & & ICACIONES & MODO & OPORTUNIDAD & INDICACIONES & MODO \\
\hline \multirow{5}{*}{7} & & $\begin{array}{r}\text { Pre-contacto } \\
\text { con el paciente }\end{array}$ & \multirow{5}{*}{$\underset{\text { Frotado }}{\square}$} & \multirow{5}{*}{8} & $\begin{array}{r}\text { Pre-contacto } \\
\text { con el paciente }\end{array}$ & \multirow{5}{*}{$\begin{array}{l}\square \\
\text { Frotado } \\
\square \\
\text { Lavado }\end{array}$} \\
\hline & & $\begin{array}{l}\text { Pre-tarea } \\
\text { aséptica }\end{array}$ & & & $\begin{array}{l}\text { Pre-tarea } \\
\text { aséptica }\end{array}$ & \\
\hline & & $\begin{array}{l}\text { Post-contacto } \\
\text { dos corporales }\end{array}$ & & & $\begin{array}{r}\text { Post-contacto } \\
\text { fluídos corporales }\end{array}$ & \\
\hline & & $\begin{array}{l}\text { Post-contacto } \\
\text { con el paciente }\end{array}$ & & & $\square \quad \begin{array}{r}\text { Post-contacto } \\
\text { con el paciente }\end{array}$ & \\
\hline & & $\begin{array}{l}\text { Post-contacto } \\
\text { con el entorno }\end{array}$ & & & $\square \begin{array}{r}\text { Post-contacto } \\
\text { con el entorno }\end{array}$ & \\
\hline
\end{tabular}


ANEXO 3

1.- Bundle CVC (paquete de medidas). Lista de chequeo para la inserción de CVC

\begin{tabular}{|c|c|c|c|c|c|c|c|c|c|}
\hline \multicolumn{10}{|l|}{ HOSPITAL } \\
\hline \multicolumn{2}{|c|}{$\begin{array}{c}\text { BUNDLE - CVC } \\
\text { Paquete de Medidas } \\
\end{array}$} & \multicolumn{8}{|c|}{ INSERCIÓN DE CATÉTERES VENOSOS CENTRALES } \\
\hline \multicolumn{5}{|c|}{ Fecha: .......... / _........... / .......... } & \multicolumn{5}{|l|}{ UCI: } \\
\hline \multicolumn{5}{|c|}{ HISTORIA CLÍNICA Nº: } & \multicolumn{2}{|c|}{ OBSERVADOR } & & & \\
\hline \multirow{2}{*}{\multicolumn{2}{|c|}{$\begin{array}{l}\text { OPERADOR } \\
\text { (el que coloca el CVC) } \\
\text { (INICIALES) }\end{array}$}} & MED & & & \multirow{2}{*}{$\begin{array}{l}\text { AYUDANTE } \\
\text { (INICIALES) }\end{array}$} & \multirow{2}{*}{\begin{tabular}{|l|} 
MED \\
ENF \\
\end{tabular}} & & & \\
\hline & & ENF & & & & & & & \\
\hline \multicolumn{10}{|c|}{ HIGIENE DE MANOS } \\
\hline \multirow{2}{*}{ OPERADOR } & M1 & \multicolumn{4}{|c|}{ ANTES DEL CONTACTO CON EL PACIENTE } & Sí & & No & \\
\hline & M2 & \multicolumn{4}{|c|}{$\begin{array}{l}\text { ANTES DE REALIZAR UNA TAREA ASÉPTICA } \\
\text { (Colocar el CVC) }\end{array}$} & Sí & & No & \\
\hline AYUDANTE & M1 & \multicolumn{4}{|c|}{ ANTES DEL CONTACTO CON EL PACIENTE } & Sí & & No & \\
\hline \multicolumn{6}{|c|}{$\begin{array}{l}\text { 2. KIT O BANDEJA CONTENIENDO TODOS JUNTOS LOS ELEMENTOS } \\
\text { NECESARIOS PARA LA INSERCION DEL CVC }\end{array}$} & Sí & & No & \\
\hline \multicolumn{6}{|c|}{$\begin{array}{l}\text { 3. ANTISEPSIA ADECUADA DEL SITIO DE INSERCION: } \\
{ }^{*} \text { CON CLORHEXIDINA SOLUCION AL } 2 \% \text { O } \\
\text { CLORHEXICINA EN BASE ALCOHOLICA }\end{array}$} & Sí & & No & \\
\hline \multicolumn{6}{|c|}{$\begin{array}{l}\text { *APLICACIÓN MEDIANTE FROTADO SOBRE LA PIEL DURANTE UN } \\
\text { MÍNIMO DE } 30 \text { SEGUNDOS Y UN MÁXIMO DE } 2 \text { MINUTOS }\end{array}$} & Sí & & No & \\
\hline \multicolumn{6}{|c|}{$\begin{array}{l}\text { *TIEMPO DE ESPERA (1-2 minutos aprox.) PARA SECADO } \\
\text { DEL ANTISEPTICO ANTES DE PUNZAR LA PIEL }\end{array}$} & Sí & & No & \\
\hline \multicolumn{6}{|c|}{$\begin{array}{l}\text { 4. USO DE BARRERAS MAXIMAS DE PRECACUCION: } \\
\text { PARA EL PACIENTE: COBERTURA COMPLETA } \\
\text { CON COMPRESA GRANDE O SABANA ESTERIL }\end{array}$} & Sí & & No & \\
\hline \multicolumn{10}{|c|}{ MAXIMAS DE PRECAUCIÓN: PARA EL OPERADOR } \\
\hline \multirow{2}{*}{$\begin{array}{c}\text { USO DE } \\
\text { CAMISOLÍN } \\
\text { ESTERIL } \\
\text { OPERADOR }\end{array}$} & Sí & & \multirow{2}{*}{$\begin{array}{l}\text { USO DE } \\
\text { CAMISOLÍN } \\
\text { ESTERIL } \\
\text { AYUDANTE }\end{array}$} & Sí & & \multicolumn{4}{|c|}{ COMENTARIOS } \\
\hline & No & & & No & & & & & \\
\hline \multirow{2}{*}{$\begin{array}{l}\text { USO DE GORRO } \\
\text { OPERADOR }\end{array}$} & Sí & & \multirow{2}{*}{$\begin{array}{l}\text { USO DE GORRO } \\
\text { AYUDANTE }\end{array}$} & Sí & & \multicolumn{4}{|c|}{ COMENTARIOS } \\
\hline & No & & & No & & & & & \\
\hline \multirow{2}{*}{$\begin{array}{l}\text { USO DE BARBIJO } \\
\text { OPERADOR }\end{array}$} & Sí & & \multirow{2}{*}{$\begin{array}{l}\text { USO DE BARBIJO } \\
\text { AYUDANTE }\end{array}$} & Sí & & \multicolumn{4}{|c|}{ COMENTARIOS } \\
\hline & No & & & No & & & & & \\
\hline \multirow{2}{*}{$\begin{array}{l}\text { USO DE GUANTES } \\
\text { ESTERILES } \\
\text { OPERADOR }\end{array}$} & Sí & & \multirow{2}{*}{$\begin{array}{l}\text { USO DE GUANTES } \\
\text { ESTÉRILES } \\
\text { AYUDANTE }\end{array}$} & Sí & & $\mathrm{DE}$ & SER & $\begin{array}{ll}\mathrm{NCA} \\
\mathrm{CCES}\end{array}$ & $\begin{array}{l}\mathrm{O} \\
\mathrm{RIO}\end{array}$ \\
\hline & No & & & No & & & COME & TAR & \\
\hline
\end{tabular}




\section{ANEXO 4}

\section{Actividades que se realizaron durante el programa}

Medidas

1. Programa de vigilancia

\section{Programa de capacitación}

a. Charlas para capacitar a cada grupo de enfermeros de cada turno (en especial, al nuevo entrante), plantel médico, junto con el jefe de turno y el supervisor de enfermería sobre el nuevo paquete de medidas recomendadas para el control de infecciones asociadas a catéteres en la Unidad, que se realizó cada 6 meses.

b. Carteles informativos dentro de la Unidad para el personal de salud.

c. Carteles informativos para padres acerca de las medidas de higiene antes del ingreso a la Unidad.

d. Disponibilidad de solución de alcohol en gel fraccionado o clorhexidina jabonosa para cada Unidad.

\section{Protocolo de colocación y manejo de catéteres}

a. Técnica de colocación de catéteres centrales: las barreras para la colocación del catéter central deben incluir uso de gorro, barbijo, camisolín, guantes y campos estériles en un kit prearmado con lista de chequeo y respetando los tiempos de espera al aplicar un antiséptico específico (clorhexidina al $2 \%$ u otro) y lograr su máximo efecto antes de la inserción del CVC.

b. No se debe cambiar en forma rutinaria el catéter central en plazos preestablecidos.

c. El recambio por técnica de Seldinger solo se realizará si no existe infección en el sitio de entrada o bacteriemia asociada a catéter en esa posición y únicamente en circunstancias excepcionales.

d. Discusión diaria de la necesidad de la vía central: no se debe prolongar innecesariamente el uso de catéteres.

\section{Uso y cuidado de los catéteres centrales}

- Se debe proteger el sitio de inserción del catéter con apósito estéril, transparente, semipermeable, permeable o gasa seca.

- Se debe cambiar el sistema de protección solo si está sucio o mojado.

- La frecuencia de curaciones del sitio de inserción debe realizarse de acuerdo con la evaluación local o cada 7 días. - Se deben limpiar con gasa embebida en alcohol los tapones de las llaves de 3 vías por 15 segundos antes del acceso a los sistemas de infusión.

- Se debe asegurar la ausencia de roturas o filtraciones en el sistema de infusión y mantener el circuito sellado cuando no esté en uso.
Responsable

Servicio de Infectología y Epidemiología.

Supervisor de enfermería junto con jefes de cada turno.

Servicio de Infectología y Epidemiología.

Servicio de Infectología y Epidemiología.

Servicio de Infectología y Epidemiología.

Servicio de Infectología y Epidemiología.

Farmacia.

Enfermera link. *

Plantel médico.

Enfermera link.

Otros miembros del equipo de salud.

Plantel médico.

Enfermera link.

Otros miembros del equipo de salud.

Plantel médico.

Enfermera link.

Otros miembros del equipo de salud.

Plantel médico.

Enfermera link.

Otros miembros del equipo de salud.

Plantel médico.

Enfermera link.

Encargado de turno.

Otros miembros del equipo de salud.

* Enfermera link: enfermera de Terapia Intensiva. No recibe pacientes a cargo; cumple todo su horario dentro de la Unidad como enfermera en control de infecciones. 


\section{Fases de implementación del programa}

\section{Fases del programa}

Fase I: Control histórico

Agosto de 2010-mayo de 2011. Estadística previa de cada centro participante.

Fase II: Diseño y aplicación de la intervención - Capacitación y entrenamiento del personal elegido (un enfermero y un médico) de cada unidad participante para la implementación del programa a cargo del centro coordinador.

- Entrega de CD con todas las herramientas (instructivos, planillas) para llevar a cabo el programa.

- Entrenamiento del personal de salud a cargo del personal que se capacitó en el centro coordinador. - Apertura de un sitio en el campus virtual del hospital coordinador con toda la información necesaria, así como asesoramiento a través de este medio.

- Aplicación de checklist de higiene de manos y de colocación de CVC.

- Monitoreo de la adherencia de higiene de manos, colocación de CVC y de la estadística de las bacteriemias de cada unidad usando las planillas correspondientes para cada actividad. 